\title{
Revista Brasileira de Enfermagem REBEn \\ Programa educativo sobre sexualidade e DST: relato de experiência com grupo de adolescentes
}

\author{
Educative program about sexuality and Sexually Transmitted Diseases: \\ experience report with a group of adolescents \\ Programa educativo sobre la sexualidad y Enfermedades Sexualmente \\ Transmisibles: relacto de experiencia con un grupo de adolescentes
}

\section{Márcia M. Souza}

Professora Assistente e Pesquisadora do NUCLAIDS (Núcleo de Ações Interdisciplinares em DST e AIDS)/FEN/ Universidade Federal de Goiás, Goiânia, GO.

Endereco para contato:

Rua das Quaresmeiras Qd.24 Lt.10, Condomínio Aldeia do Vale. Goiânia, GO -

CEP: 74680450

marcia@fen.ufg.br

Sandra Brunini

Professora Assistente e Pesquisadora do NUCLAIDS/FEN/Universidade Federal de Goiás, Goiânia, GO.

Nilza A. M. Almeida

Professora Assistente e Pesquisadora do NUCLAIDS/FEN/Universidade Federal de Goiás, Goiânia, GO.

Denize B. Munari

EProfessora Titular e Pesquisadora do NEPSI/FEN/Universidade Federal de Goiás,

Goiânia, GO

Fonte Financiadora: Coordenação Nacional de DST/AIDS - UNESCO

Submissão: $18 / 05 / 2006$

Aprovação: 21/12/2006

\section{RESUMO}

O objetivo deste estudo foi relatar a experiência com grupo de adolescentes através de oficinas vivenciais sobre educação sexual. Realizamos 10 oficinas com adolescentes de baixa renda do Município de Aparecida de Goiânia/GO, que aconteceram a partir de reflexões e socialização do conhecimento sobre esta temática. 0 trabalho foi embasado na metodologia participativa de Paulo Freire e potencializado pela atenção dos coordenadores ao movimento do grupo, considerando suas necessidades e possibilidades. Concluímos que para o sucesso do trabalho interativo o coordenador de grupos deve ter uma escuta sensivel aos anseios do grupo, especialmente o de adolescente. É necessário estimular a participação para a aquisição de conhecimentos o que favorecerá o exercício da cidadania e transformação da sua realidade social.

Descritores: Saúde do adolescente; Promoção da saúde; Grupos de risco; Doenças SexualmenteTransmissíveis.

\section{ABSTRACT}

The object of this study was to give an account of the experiment with a teenage group by using sexual education experiences of their own. Ten workshops were made with low-income teenagers of Municipio de Aparecida de Goiânia /GO, which happened because of the socialization and reflections about the contents of this study. The work was based on Paulo Freire's Participative Methodology and made better by the attention of the coordinators to every single group, considering their special needs and possibilities. We conclude that for the success of this work, the coordinator must listen to the group with a very sensitive prospect, especially when dealing with a teenage group. It is necessary to stimulate the participation, so that more can be learnt and the citizen work can be able to change its social reality. Descriptors: Adolescent health; Health promotion; Risk groups; Sexually Transmitted Diseases.

\section{RESUMEN}

El objetivo de este estudio fue relatar la experiencia con grupo de adolescentes a través de talleres vivenciales sobre educación sexual. Realizamos 10 talleres con adolescentes de bajo poder adquisitivo del Municipio de Aparecida de Goiânia-GO, que se dieron a partir de reflexiones y socialización del conocimiento sobre esta temática. El trabajo fue realizado en base a la metodología participativa de Paulo Freire y potenciado por la atención de los coordinadores del movimiento del grupo, considerando sus necesidades y posibilidades. Concluimos que para el éxito del trabajo interactivo el coordinador de grupos debe tener una escucha sensible a los anhelos del grupo, especialmente de los adolescentes. Es necessário estimular la participación para la adquisición de nuevos conocimientos favoreciendo asi el ejercicio de la ciudadanía para transformación de su realidad social.

Descriptores: Salud del Adolescente; Promoción de la salud; Grupos de riesgo; Enfermedades Sexualmente transmisibles.

Souza MM, Brunini S, Almeida NAM, Munari DB. Programa educativo sobre sexualidade e DST: relato de experiência com grupo de adolescentes. Rev Bras Enferm 2007 jan-fev; 60(1):102-5.

\section{INTRODUÇÃO}

No que diz respeito às Doenças Sexualmente Transmissíveis (DSTs) e a Síndrome da Imunodeficiência Adquirida (Aids), é imprescindivel que a prevenção mereça enfoque prioritário, sobretudo quando o alvo das ações é a população jovem. As modificações bio-psico-sociais que ocorrem no adolescente podem interferir no processo natural do seu desenvolvimento, fazendo com que ele sinta necessidade de experimentar comportamentos que os deixem mais vulneráveis a riscos para a sua saúde, inclusive no aspecto da sexualidade.

A não adesão aos métodos contraceptivos, as medidas de prevenção para a não aquisição das DSTs, 
a curiosidade pelas drogas, bem como a necessidade de afirmação grupal, tornam os adolescentes susceptiveis à aquisição de doenças de transmissão sexual. Estudos mostram que o desconhecimento do modo de contágio das DSTs e a não importância dos métodos preventivos podem levar prejuízo a saúde sexual e reprodutiva dos adolescentes como a infertilidade, gravidez ectópica e câncer uterino, além de serem o principal fator facilitador da transmissão pela via sexual(1,2).

Doenças de transmissão sexual têm sido causa de agravos em crianças e adolescentes em todo o mundo e a população jovem é considerada a mais vulnerável às DSTs, principalmente à infecção pelo HIVIAids ${ }^{(3,4)}$.

No Brasil, estima-se que a cada ano, quatro milhões de jovens tornamse sexualmente ativos e que ocorram cerca de 12 milhões de DST ao ano, das quais, um terço em indivíduos com menos de 25 anos $^{(5)}$. Considerando o longo período de latência da infecção pelo HIV/Aids ${ }^{(6)}$, estes dados sugerem que a infecção ocorra, provavelmente, na adolescência.

A educação em saúde é uma ação básica cujo objetivo é capacitar indivíduos e/ou grupos para assumirem ou ajudarem na melhoria das suas condições de saúde ${ }^{(7)}$. Nesse sentido, os grupos têm sido utilizados como um instrumento valioso e até mesmo imprescindível para o alcance deste objetivo. Por definição, o grupo é uma unidade de duas ou mais pessoas empenhadas em recíproca interação psicológica, onde os membros podem estar no mesmo ambiente ou distantes fisicamente, mas interagindo através de algum meio de comunicação(8).

O Ministério da Saúde recomenda que tecnologias de grupo sejam utilizadas nas atividades com adolescentes, como estratégias de intervenção para as atividades de educação em saúde, principalmente no contexto da epidemia da Aids e outras DSTs ${ }^{(9,10)}$.

O grupo, para o adolescente, constitui-se em um espaço para formação de uma nova identidade, ainda que intermediária entre a família e a sociedade, em que ele pode experimentar e exercer novos papéis. Os indivíduos, principalmente os jovens, quando não estão em grupos se sentem expostos e inseguros, mas quando estão agrupados se sentem confiantes quanto aos valores delimitados de seus pares, pois diluem sentimentos de vergonha, medo, culpa ou até mesmo inferioridade ${ }^{(11)}$.

Alguns estudos apontam para a necessidade de adoção de práticas educativas mais eficientes e mais precocemente, principalmente no ambiente escolar, devido a alguns resultados encontrados como os limites de informações ou conhecimento insuficiente e errôneo sobre as práticas preventivas quanto a aquisição das DSTs, associado a isso o baixo nível de escolaridade, e ainda um sistema educacional desestimulante o qual tem o dever de levar a informação correta até o público alvo e permitir sua participação no processo de ensino aprendizagem ${ }^{(12-15)}$.

Em um estudo prévio, verificou-se uma grande vulnerabilidade às doenças de transmissão sexual em adolescentes escolares de uma região do entorno de Goiânia, Goiás. A média de idade da primeira relação sexual foi de 14,9 anos, $44,1 \%$ dos indivíduos referiram uso irregular ou não do preservativo e uma média de quatro parceiros ${ }^{(16)}$.

Este cenário nos motivou a realizar um projeto de educação em saúde para esta população. Neste artigo objetivamos relatar a experiência de educação em saúde com adolescentes, com enfoque em sexualidade e prevenção de DSTs e Aids, utilizando-se tecnologia de grupo.

\section{CAMPO DA EXPERIÊNCIA}

O Município de Aparecida de Goiânia está localizado no entorno de Goiânia, Goiás, e possui 417.409 habitantes. Tem uma grande afluência migratória, com altos índices de violência urbana, e em algumas regiões periféricas a população apresenta-se com baixo nível socioeconômico e educacional, ou seja, características típicas de cidades circunvizinhas à metrópole.

Diante deste contexto social e de saúde o Núcleo de Ações Interdisciplinares em DST/HIV/Aids (NUCLAIDS), da Faculdade de
Enfermagem da Universidade Federal de Goiás (FEN/UFG), cadastrado no diretório de Grupos de Pesquisas do CNPq, em extensão às suas atividades, tem realizado projetos de capacitação de recursos humanos com enfoque na prevenção das DSTs para profissionais do Programa de Saúde da Família (PSF) do referido município.

Assim, O NUCLAIDS elaborou um projeto de extensão intitulado "EDUCAR É PREVENIR", o qual foi financiado pelo Ministério da Saúde/ UNESCO, através do Programa Nacional de DST/Aids. Nesse projeto foram oferecidos distintamente cursos de capacitação para enfermeiros e agentes comunitários de saúde do PSF, durante os quais os participantes identificaram a necessidade de se trabalhar com os adolescentes daquele município, através de um trabalho educativo de prevenção e sensibilização sobre questões relacionadas à sexualidade, prevenção de DSTs/HIV/ Aids e gravidez.

A fim de atender àquela demanda e com base em informações prévias sobre a população de adolescentes daquela região, bem como nas experiências do NUCLAIDS/FEN/UFG elaborou-se um projeto de continuidade para desenvolver as atividades propostas pelos profissionais do Programa de Saúde da Família da região. Este programa tem uma característica de mudança no novo modelo assistencial a saúde, e que exige dos profissionais da equipe multidisciplinar uma atenção sistematizada e um manejo específico para trabalhar com grupos de adolescentes que como já sabemos é permeada por peculiaridades e especificidades nesta faixa etária ${ }^{(3,15,17) .}$

\section{ESTRUTURA E FUNCIONAMENTO DAS OFICINAS}

No projeto de continuidade, também financiado pelo Ministério da Saúde/ UNESCO, foram realizadas dez oficinas de prevenção de DSTs. Cada uma delas funcionou com quatro horas de duração e quinze participantes por oficina, num total de cento e cinqüenta pessoas atendidas. $O$ objetivo foi sensibilizar os adolescentes atendidos pelas equipes de PSF de Aparecida de Goiânia para o exercício da cidadania, da sexualidade saudável e para a prevenção das DST/HIV.

Para tanto, desenvolvemos com aquela população um programa de atividades, conforme descrito no Quadro1, que contemplou técnicas grupais e vivências específicas para oportunizar as discussões dos temas propostos como: 1) Cuidados com o corpo; 2) Gênero e Sexualidade; 3) Ética, direitos humanos e cidadania; 4) Como evitar DSTs/HIV e gravidez.

No início das atividades foram utilizadas técnicas de integração para a apresentação dos membros do grupo e posteriormente houve a pactuação do contrato de trabalho. Na operacionalização das oficinas participaram cinco enfermeiros docentes da FEN/UFG que exerceram papel de mediadores, visando à construção do conhecimento sobre sexualidade e prevenção de DSTs/ AIDS e gravidez, denominados de coordenadores. As oficinas aconteceram no mês de agosto de 2003 e foram executados nos espaços físicos dos centros comunitários, escolas e igrejas da comunidade, previamente contactados pelos agentes de saúde do PSF.

O modelo pedagógico fundamentou-se na educação conscientizadora/ problematizadora de Paulo Freire, sustentada pela metodologia participativa e dialogada que favorece uma relação crítica e transformadora ${ }^{(18)}$. O cronograma das atividades teve foco nos temas geradores de fonte problematizadora e motivadora e as atividades foram desenvolvidas de forma dinâmica a partir de situações, reflexões e verbalizações de experiências vividas além de dramatizações. Como recursos didáticos foram utilizados bonecos, próteses dos órgãos genital feminino e masculino, figuras, cartazes, músicas, fotos e desenhos.

\section{OENFOQUE GRUPAL NAABORDAGEM DOS ADOLESCENTES}

É necessário que se elabore um planejamento detalhado no qual sejam consideradas as necessidades da população que se pretende trabalhar. Quando 


\begin{tabular}{|c|c|c|c|}
\hline \multicolumn{4}{|c|}{$\begin{array}{l}\text { População Alvo: Adolescentes residentes nas áreas de abrangência do Prog } \\
\text { Equipe de facilitadores: Docentes da Faculdade de Enfermagem da UFG. }\end{array}$} \\
\hline \multicolumn{4}{|c|}{$\begin{array}{l}\text { Objetivo Geral: Os adolescentes deverão ser capazes de compreender as temáticas e adotar práticas saudáveis visando à melhoria da qualidade de vida, o exercício da } \\
\text { cidadania e uma participação transformadora da realidade social. }\end{array}$} \\
\hline Objetivos específicos & Temas Geradores & Estratégias & Recursos \\
\hline $\begin{array}{l}\text { - Compreender os cuidados básicos } \\
\text { com o próprio corpo; } \\
\text { - Assumir práticas e hábitos saudáveis. }\end{array}$ & Cuidados com o corpo & $\begin{array}{l}\text { - Confecção de cartazes com desenho do corpo } \\
\text { para apresentação em grupo; } \\
\text { - descrever quais os cuidados cada um dispensa } \\
\text { ao próprio corpo. }\end{array}$ & - Papel manilha, pincel atômico, fita crepe. \\
\hline $\begin{array}{l}\text { - Diferenciar sexo e sexualidade; } \\
\text { - Citar as diferenças entre o gênero } \\
\text { feminino e masculino; } \\
\text { - Diferenciar a anatomia dos órgãos } \\
\text { genitais femininos e masculinos; } \\
\text { - Identificar as diferentes práticas } \\
\text { sexuais e riscos para a saúde. }\end{array}$ & Gênero e Sexualidade & $\begin{array}{l}\text { - Solicitação do entendimento do adolescente } \\
\text { sobre sexo e sexualidade, a diferença entre } \\
\text { homem e mulher e as práticas sexuais; } \\
\text { - Aula expositiva dialogada sobre sexo, } \\
\text { sexualidade, menstruação e masturbação. }\end{array}$ & $\begin{array}{l}\text { - Figuras e próteses do órgão genital masculino e } \\
\text { feminino; } \\
\text { - Papel manilha, pincel atômico, fita crepe. }\end{array}$ \\
\hline $\begin{array}{l}\text { - Refletir sobre as questões éticas do } \\
\text { cidadão, seus direitos e deveres; } \\
\text { - Compreender a sua importância } \\
\text { social como cidadão adolescente. }\end{array}$ & $\begin{array}{l}\text { Ética, direitos humanos e } \\
\text { cidadania }\end{array}$ & $\begin{array}{l}\text { - Exposição de filme educativo sobre cidadania; } \\
\text { - Discussão em grupo sobre o filme apresentado }\end{array}$ & - Televisão, videocassete, fita VHS. \\
\hline $\begin{array}{l}\text { - Conceituar as DSTs/Aids; } \\
\text { - Conhecer os modos de prevenção e } \\
\text { transmissão DSTs/Aids; } \\
\text { - Distinguir os sinais e sintomas de } \\
\text { DSTs/Aids }\end{array}$ & $\begin{array}{l}\text { Como evitar DSTs e } \\
\text { Aids? }\end{array}$ & $\begin{array}{l}\text { - Exposição em cartazes sobre o conhecimento } \\
\text { de DSTs; } \\
\text { - Aulas expositivas dialogadas sobre as principais } \\
\text { DSTs. }\end{array}$ & $\begin{array}{l}\text { - Papel manilha, pincel atômico, fita crepe, cola, } \\
\text { revistas e tesouras. } \\
\text { - Álbum seriado com figuras ilustrativas das } \\
\text { principais DSTs. }\end{array}$ \\
\hline - Conhecer os métodos contraceptivos & Como evitar gravidez? & $\begin{array}{l}\text { - Demonstração de Kit de planejamento familiar } \\
\text { contendo os contraceptivos mais conhecidos. }\end{array}$ & - Kit educativo de planejamento familiar. \\
\hline
\end{tabular}

Quadro 1. Temas geradores do Programa Educativo.

o público alvo é a população jovem, o coordenador de grupos deve ter clareza do papel que deseja desempenhar estabelecendo um vínculo de proximidade com estes indivíduos. As possibilidades de sucesso no trabalho com grupos de adolescentes dependem, e muito, da relação de confiança que deve existir entre coordenador e adolescentes ${ }^{(3,15)}$.

A todo o momento exigiu-se do coordenador uma postura de compromisso, afetividade, comunicação adequada e clara. Possivelmente, esses movimentos facilitaram uma maior interação entre os membros envolvidos, promovendo, assim momentos de espontaneidade para as trocas de experiências e discussões. 0 conhecimento da dinâmica de grupo nesse contexto facilita a percepção dos movimentos de abertura e resistência do grupo, inerentes ao processo grupal|(19). Nesse caso 0 coordenador de grupos deve ter sabedoria suficiente para respeitar os tempos internos e externos dos participantes do grupo, de modo a potencializar o grupo para o seu melhor desempenho e o favorecimento da ação dos efeitos como os descrevem.

\section{ANALISANDO O PROCESSO DO GRUPO}

No processo de desenvolvimento das oficinas foi percebida uma interação mútua e afetiva com muita integração entre os coordenadores e os grupos de adolescentes, favorecendo o bom desenvolvimento das ações. As atividades foram realizadas de forma dinâmica a partir de situações, reflexões, verbalizações de experiências vividas e dramatizações, onde se priorizou 0 aprendizado grupal, ou seja, o potencial das trocas de experiências.

À medida que foram realizadas as atividades, os adolescentes percebiam a importância da transformação da realidade bem como da mudança do comportamento para hábitos saudáveis e atitudes positivas. Esses resultados também foram encontrados nos trabalhos de Dias e Bueno ${ }^{(20)}$, os quais referem que a aquisição de novos conhecimentos, habilidades e atitudes adotadas pelo adolescente resultam na melhoria da sua qualidade de vida, permitindo o resgate da cidadania e uma participação transformadora da realidade social.

Em relação ao movimento grupal, observou-se a coesão como um fenômeno mais constante. Fenômenos como a coesão e a resistência são comuns por parte dos membros de um grupo. Para Castilho(21), a coesão pode contribuir para um alto nível de produção para as mudanças, mas também poderá ser uma forte variável de resistência às modificações. Os indivíduos quando em grupo podem expressar resistências as mudanças e isso exige do facilitador uma grande habilidade em lidar com essas situações. Finalmente, leva o grupo a superar possíveis resistências.

O movimento grupal relacionado aos temas ou tarefas foi caracterizado principalmente pela coesão, o que favoreceu a potencialização do grupo e 0 alcance dos objetivos propostos. Esse movimento foi caracterizado pela interação mútua entre os adolescentes e entre estes e os coordenadores, assim como a presença de equilíbrio e ligação recíproca. Acreditamos que este resultado ocorreu, possivelmente, pela homogeneidade do grupo (faixa etária, classe social, vulnerabilidade a riscos às DSTs/AIDS, em fase reprodutiva), mas também pela utilização de uma abordagem educativa problematizadora que considerou o movimento do grupo, suas necessidades e possibilidades.

Na avaliação final das oficinas foi observado pelos docentes/facilitadores que cada grupo de adolescentes expressou uma forma diferente de se envolver e participar nas atividades, o que já é esperado e considerado inerente a cada indivíduo.

Ressalta-se que em relação às técnicas e estratégias grupais utilizadas é importante considerar que para a adequação das mesmas aos objetivos propostos, o facilitador deve considerar o movimento que o próprio grupo de adolescente mostra, além de respeitar e valorizar os seus conhecimentos e estimular a socialização das experiências ${ }^{(17,22,23)}$.

Finalmente, destaca-se que a tarefa de coordenar grupos é algo muito delicado e complexo, em particular, o grupo de adolescentes. Exige-se do coordenador uma análise e revisão dos valores e crenças, e dos sentimentos expressos que movimentam o campo grupal. Esses são fatores dinâmicos, 
que quando bem analisados e conduzidos pelo coordenador possibilitam uma melhor expressão do grupo, com conseqüente aproveitamento dos potenciais terapêuticos que dele emanam ${ }^{(22)}$.

\section{CONSIDERAÇÕES FINAIS}

A realização deste trabalho exigiu esforço, dedicação, reflexão e desafio para a execução das ações planejadas, especialmente para superar a experiência como docentes que contemplava, na maioria das vezes, 0 modelo pedagógico tradicional, considerado pouco reflexivo. Diante deste contexto, a teoria da problematização de Paulo Freire, bem como os fundamentos do trabalho grupal, possibilitaram o alcance dos objetivos propostos, considerando que esse modelo é fundamentado no diálogo aberto e reflexivo, que o educando parte de sua própria realidade para interagir, trocar experiências e adquirir novos conhecimentos.

O envolvimento contínuo como educadores e enfermeiros e o conhecimento sobre os temas específicos, a dinâmica de grupo, acrescida da credibilidade dispensada pelos adolescentes ao grupo de coordenadores foram pontos facilitadores para a operacionalização das oficinas e o alcance dos objetivos propostos. Como ponto dificultador, apontamos o pouco tempo destinado para abordar todas as temáticas frente à motivação e participação ativa dos adolescentes.

\section{REFERÊNCIAS}

1. Julião TC, Fernandes AFC, Gurgel AH. Prevenção de DST/ AIDS: Uma abordagem junto a famílias de adolescentes. Rev. RENE 2001;2(1):53-9.

2. Trani F, Gnisci F, Nobile CGA, Angelillo IF. Adolescents and sexually transmitted infections: Knowledge and behavior in Italy. J Paediatric Child Health 2005;41:260-4

3. Santos LV, Santos EC. Adolescentes, jovens e aids no Brasil. (citado em 18 nov 2004). Disponivel em: URL: http:// www.aids.gov.br/final/prevencao/adolescentes.htm

4. Ayres RCM. HIVIAIDS, DST e abuso de drogas entre adolescentes: vulnerabilidade e avaliação de ações preventivas São Paulo (SP): Casa de Edição. Departamento de Medicina da Faculdade de Medicina da Universidade de São Paulo; 1996.

5. Ministério da Saúde (BR). Dados e pesquisas em DST e Aids. (citado em 11 nov 2005). Disponível em: URL: http:// www.aids.gov.br/final/dados/DST.htm

6. Oliveira SHS, Dias MR, Silva MIT. Adolescentes e AIDS: Fatores que Influenciam a Intenção de Uso do Preservativo. J Bras Doenças Sex Transm 2005;17(1):32-8.

7. Kawamoto EE, Santos $\mathrm{MCH}$, Matos $\mathrm{CM}$. Enfermagem Comunitária. São Paulo (SP): EPU; 1995.

8. Andaló CSA. O Papel de Coordenador de Grupos. Psicologia USP 2001;12(1):135-52.

9. Ministério da Saúde (BR). Coordenação Nacional de DST/AIDS Manual de multiplicador: adolescente. Brasília (DF): Ministério da Saúde; 2000.

10. Ministério da Saúde (BR). Projeto de Apoio à Sustentabilidade e Gestão Estratégica das Políticas de Controle do HIV/Aids e outras Doenças Sexualmente Transmissíveis - AIDS III. Brasília (DF): Ministério da Saúde; 2002.

11. Souza MM, Borges IK, Medeiros M, Teles SA, Munari DB. A Abordagem de Adolescentes em Grupos: 0 Contexto da Educação em Saúde e Prevenção de DST. J Bras Doenças Sex Transm 2004;16(2):18-22.
12. Borges IK, Medeiros M. Representações Sociais de DST/AIDS para Adolescentes de uma Instituição de Abrigo com Experiência Pregressa de Vida nas Ruas da Cidade de Goiânia. J Bras Doenças Sex Transm 2004;16(4):43-9.

13. Ministério da Saúde (BR) Boletim Epidemiológico AIDS 2001; 14(1).

14. Brêtas JRS, Silva CV. Interesse de escolares e adolescentes sobre corpo e sexualidade. Rev Bras Enferm 2002;55(5):52834.

15. Martini JG, Bandeira AS. Saberes e práticas dos adolescentes na prevenção das doenças sexualmente transmissíveis. Rev Bras Enferm 2003;56(2):160-3.

16. Silva P, Oliveira MDS, Matos MA. Comportamentos de risco para as doenças sexualmente transmissíveis em adolescentes escolares de baixa renda. Rev Eletrônica Enferm 2005; no prelo.

17. Antunes MJM, Egry EY. O Programa de Saúde da Família e a reconstrução da atenção básica no SUS: a contribuição da enfermagem brasileira. Rev Bras Enferm 2001;54(1):98-107.

18. Freire P. Educação como Prática Libertadora. Rio de Janeiro (RJ): Paz e Terra; 1996.

19. Munari DB, Furegato ARF. Enfermagem e grupos. Goiânia (GO): AB Editora; 2003.

20. Dias A, Bueno SMV. Programa Educativo sobre Sexualidade, DST/Aids e Sexo Seguro, Desenvolvido junto aos Alunos do Curso Técnico de Enfermagem: um Relato de Experiência. Rev Nursing 2003;58(6):14-6.

21. Castilho A. A dinâmica do trabalho em grupo. Rio de Janeiro (RJ): Qualiymark; 1998.

22. Munari DB, Ribeiro V, Lopes MM. Intervenção grupal com enfoque no cuidado emocional: relato de uma experiência. Rev Bras Enferm 2002;55(4):449-51.

23. Silva ALAC, Munari DB, Lima FV, Silva WO. Atividades Grupais em Saúde Coletiva. Rev Enferm UERJ 2003;11:18-24. 